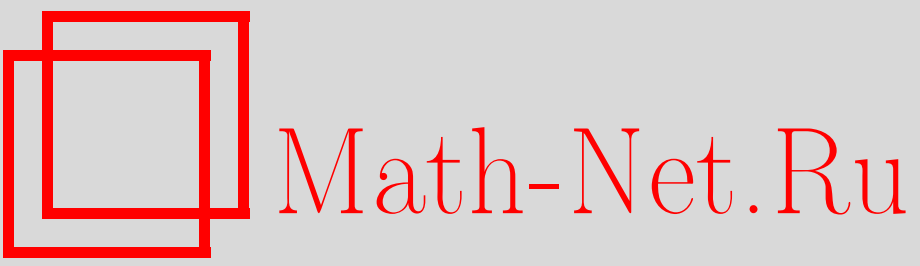

Н. Г. Мощевитин, О цепных дробях и почти арифметических прогрессиях, $У М H$, 2000, том 55, выпуск 1, 189-190

DOI: https://doi.org/10.4213/rm1623

Использование Общероссийского математического портала Math-Net.Ru подразумевает, что вы прочитали и согласны с пользовательским соглашением

http://www.mathnet.ru/rus/agreement

Параметры загрузки:

IP: 3.89 .185 .249

26 апреля 2023 г., 18:32:48 


\title{
О ЦЕПНЫХ ДРОБЯХ И ПОЧТИ АРИФМЕТИЧЕСКИХ ПРОГРЕССИЯХ
}

\author{
Н. Г. МощЕвитин
}

1. Формулировки. Почти арифметической прогрессией длины $l$ назовем множество вещественных положительных чисел $a_{1}<a_{2}<\cdots<a_{l}$, удовлетворяющее условию $\exists w>0$ : $w \leqslant a_{j+1}-a_{j} \leqslant 3 w, \forall j=1, \ldots, l-1$.

Теорема 1. Пусть натуральные числа $a_{1}, \ldots, a_{l}<q,\left(a_{j}, q\right)=1$, образуют почти арифметическую прогрессию длины $l$. Положим $a_{\nu} / q=\left[0 ; b_{\nu, 1}, \ldots, b_{\nu, s(\nu)}\right]$. Тогда найдутся индексы $\nu, \mu$ такие, что $b_{\nu, \mu} \gg l^{1 / 2}$.

Теорема 1 усиливает результат работы [1].

Теорема 2. Пусть вещественные иррациональные числа $\alpha_{1}, \ldots, \alpha_{l} \in(0 ; 1)$ образуют почти арифметическую прогрессию длины $l$. Положим $\alpha_{\nu}=\left[0 ; b_{\nu, 1}, \ldots, b_{\nu, \mu}, \ldots\right]$. Тогда найдутся индексы $\nu, \mu$ такие, что $b_{\nu, \mu} \gg l^{1 / 2}$.

Теорема 1 доказана ниже в пп. 2-4. Теорема 2 доказывается аналогично.

С.В. Конягин сообщил автору следующий пример.

Пример КонягинА. Рассматривается ряд Фарея порядка $m$, полагаем $x_{i}=10 i / m$, $i=1, \ldots,[(m-1) / 10]$. Каждое $x_{i}$ попадает в свой интервал Фарея $x_{i} \in\left[p_{1} / q_{1}, p_{2} / q_{2}\right)$. Пусть $y_{i}=\left(\sqrt{2} p_{1}+p_{2}\right) /\left(\sqrt{2} q_{1}+q_{2}\right)$. Теперь рассматривается последовательность $z_{j, i}=1 /\left(j+y_{i}\right)$, $9 m / 10<j \leqslant m, i=1, \ldots,[(m-1) / 10]$. Числа $z_{j, i}$ образуют почти арифметическую прогрессию длины $\gg m^{2}$, и вместе с тем все неполные частные имеют порядок $\ll m$. Таким образом, оценка теоремы 2 является точной по порядку.

2. Полигоны Клейна. Рассматривается плоскость $\mathbb{R}^{2}$, отнесенная к координатам $(x, y)$ и целочисленная решетка $Z^{2} \subset \mathbb{R}^{2}$. Пусть $(q ; a) \in \mathbb{Z}^{2}$ - примитивная точка, т.е. $(q, a)=1$, и пусть $q, a>0$. Рассмотрим углы $\varphi_{+}$и $\varphi_{-}$, определяемые условиями

$$
\varphi_{+}=\left\{Z=(x ; y) \in \mathbb{R}^{2}: x \geqslant 0, y \geqslant \frac{a}{q} x\right\}, \quad \varphi_{-}=\left\{Z=(x ; y) \in \mathbb{R}^{2}: y \geqslant 0, y \leqslant \frac{a}{q} x\right\} .
$$

Полигонами Клейна $\mathscr{K}_{+}(a, q)$ и $\mathscr{K}_{-}(a, q)$ будем называть те куски бесконечных ломаных $\partial\left(\operatorname{conv}\left(\varphi+\cap\left(Z^{2} \backslash\{0\}\right)\right)\right)$ и $\partial\left(\operatorname{conv}\left(\varphi-\cap\left(Z^{2} \backslash\{0\}\right)\right)\right)$, соответственно, которые состоят из конечных отрезков. (Здесь и в дальнейшем $\operatorname{conv}(\Omega)$ обозначает выпуклую оболочку множества $\Omega$.) Определим область $\Delta(a, q)$, расположенную между $\mathscr{K}_{+}(a, q)$ и $\mathscr{K}_{-}(a, q)$ :

$\Delta(a, q)=\left\{Z=(x, y) \in \mathbb{R}^{2}: x>0, y>0, Z \notin \operatorname{conv}\left(\varphi_{+} \cap\left(\mathbb{Z}^{2} \backslash\{0\}\right)\right), Z \notin \operatorname{conv}\left(\varphi-\cap\left(\mathbb{Z}^{2} \backslash\{0\}\right)\right)\right\}$. Следующий факт известен в геометрической теории цепных дробей (см. [2] и [3; c. 101]).

ЛЕмма 1. 1. Вериинами $\mathscr{K}_{-}(a, q)$, отличными от точки $(q ; a)$, являются челье точки $\left(q_{2 \mu}, p_{2 \mu}\right)$, где $q_{2 \mu}$ и $p_{2 \mu}$ суть знаменатель и числитель подходящей $\kappa$ a/q дроби с четным номером $2 \mu$.

2. Вериинами $\mathscr{K}_{+}(a, q)$, отличныци от точки $(q ; a)$, являются целье точки $\left(q_{2 \nu+1}\right.$, $\left.p_{2 \nu+1}\right)$, где $q_{2 \nu+1}$ и $p_{2 \nu+1}$ суть знаменатель и числитель подходящей $\kappa$ a/q дроби $c$ нечетным номером $2 \nu+1$.

3. Eсли $(u ; v) \in\left(\mathscr{K}_{+}(a, q) \cup \mathscr{K}_{-}(a, q)\right) \cap \mathbb{Z}^{2}-$ челая точка, то и и v суть знаменатель и числитель либо подходящей, либо промежуточной дроби для $a / q$.

4. $\Delta(a, q) \cap Z^{2}=\varnothing$.

3. Леммы. Рассмотрим области

$$
\begin{aligned}
& \Omega(a, q ; \sigma)=\left\{(x ; y) \in \mathbb{R}^{2}: 1 \leqslant x \leqslant q-1 ;\left|y-\frac{a}{q} x\right| \leqslant \frac{1}{\sigma x}\right\} \\
& \cup\left\{(x ; y) \in \mathbb{R}^{2}: 0<x<1 ;\left(\frac{a}{q}-\frac{1}{\sigma}\right) x \leqslant y \leqslant\left(\frac{a}{q}+\frac{1}{\sigma}\right) x\right\}, \quad(a, q)=1, \sigma>0, \\
& \tau(i, j ; t)=\left\{(x ; y) \in \mathbb{R}^{2} ; 0<x<t ; \frac{i x}{q}<y<\frac{j x}{q}\right\}, i<j \text { - целье }, t>0 .
\end{aligned}
$$


Следуюшая лемма модифицирует лемму 3 из [1].

ЛЕмма 2. Пусть числа $a_{0}, \ldots, a_{l}$ образуют почти арифметическую прогрессию с разностью $w \geqslant 1$. Тогда

$$
\tau\left(a_{0}, a_{l} ; t^{*}\right) \subset \bigcup_{\nu=0}^{l} \Omega\left(a_{\nu}, q ; k+2\right), \quad \text { əде } \quad t^{*} \asymp\left(\frac{q}{w(k+2)}\right)^{1 / 2} .
$$

ЛЕмма 3. Пусть неполные частные несократимой дроби а/q ограничены величиной $k$ и пусть $t^{*} / 4 \geqslant 2$. Тогда найдется челая точка

$$
Z=\left(x^{*}, y^{*}\right) \in\left(\mathscr{K}_{-}\left(a_{0}, q\right) \cup \mathscr{K}_{+}\left(a_{l}, q\right)\right) \cap \mathbb{Z}^{2}
$$

maкая, что $t^{*} / 4 \leqslant x^{*} \leqslant t^{*} / 2$.

Для доказательства леммы 3 надо рассматреть разделенный (относительно прямых $y=$ $\left(a_{0} / q\right) x$ и $\left.y=\left(a_{2} / q\right) x\right)$ параллелограмм [2], натянутый на векторы $O Z_{+}, O Z_{-}$, где

$$
Z_{+}=\left(x_{+}, y_{+}\right) \in \mathscr{K}_{+}\left(a_{l}, q\right) \cap \mathbb{Z}^{2}, \quad Z_{-}=\left(x_{-}, y_{-}\right) \in \mathscr{K}_{-}\left(a_{0}, q\right) \cap \mathbb{Z}^{2}
$$

с максимально возможным значением $\min \left\{x_{+}, x_{-}\right\}$.

Из разделенности параллелограмма, фигурировавшего в лемме 3 , вытекает

СледСтвиЕ. Точка $\left(x^{*}, y^{*}\right)$ - общая точка для полигонов $\mathscr{K}_{\sigma}\left(a_{0}, q\right), \mathscr{K}_{\sigma}\left(a_{l}, q\right)$, где $\sigma=+$ или - .

ЛЕмма 4. Пусть все неполные частные числа $а / q$ ограничены величиной $k$. Пусть $(x, y)$ - челая точка на одном из полигонов $\mathscr{K}_{ \pm}(a, q)$. Тогда $|(a / q) x-y|<(k+1) / x$.

ДокАЗАТЕльство. $y / x$-подходящая или промежуточная дробь для $a / q$. Если это подходящая дробь, то $|(a / q) x-y|<1 / x$, и все доказано. Если же промежуточная, то $q_{\nu}<x=q_{\nu}+$ $b q_{\nu+1}<q_{\nu+2} \leqslant(k+1) q_{\nu+1}$, ибо $1 \leqslant b \leqslant k-1$. Имеем $|(a / q) x-y|<\left|(a / q) q_{\nu}-p_{\nu}\right|<$ $1 / q_{\nu+1}<(k+1) / x$. Лемма доказана.

4. Доказательство теоремы 1 . В силу следствия леммы 3 и леммы 4 имеем

$$
\left(a_{l}-a_{0}\right) t^{*} / 4 q<\max \left\{\left|\left(a_{l} / q\right) x^{*}-y^{*}\right|,\left|\left(a_{0} / q\right) x^{*}-y^{*}\right|\right\}<(k+1) / x^{*} \leqslant 4(k+1) / t^{*} .
$$

Теперь получается, что $k \gg\left(t^{*}\right)^{2} l w / q \gg(q / w k) l w / q=l / k$. Итак, $k^{2} \gg l$, и теорема доказана.

Настоящая работа выполнена при финансовой поддержке Российского фонда фундаментальных исследований (гранты №№ 99-01-00357 и 00-15-96109) и фонда EFAKOD.

\section{СПИСОК ЛИТЕРАТУРЫ}

[1] Мощевитин Н. Г. // Диофантовы приближения. Математические записки. Т. 2, 1996. С. 99-109. [2] Делоне Б. Н. // Изв. АН СССР. Сер. матем. 1947. Т. 11. №6. С. 505-538. [3] Erdös P., Gruber P. M., Hammer J. Lattice Points. Harlow/New York: Longman/ Willey, 1989.

Московский государственный 